\title{
Ordinary and degenerate Euler numbers and polynomials
}

\author{
Taekyun Kim ${ }^{1,2}$, Dae San Kim³ ${ }^{3}$ Han Young Kim² and Jongkyum Kwon ${ }^{4 *}$
}

\section{"Correspondence:}

mathkjk26@gnu.ac.kr

${ }^{4}$ Department of Mathematics

Education and ERI, Gyeongsang

National University, Jinju, Republic of Korea

Full list of author information is

available at the end of the article

\begin{abstract}
In this paper, we study some identities on Euler numbers and polynomials, and those on degenerate Euler numbers and polynomials which are derived from the fermionic $p$-adic integrals on $\mathbb{Z}_{p}$. Specifically, we obtain a recursive formula for alternating integer power sums and representations of alternating integer power sum polynomials in terms of Euler polynomials and Stirling numbers of the second kind, as well as various properties about Euler numbers and polynomials. In addition, we deduce representations of degenerate alternating integer power sum polynomials in terms of degenerate Euler polynomials and degenerate Stirling numbers of the second kind, as well as certain properties on degenerate Euler numbers and polynomials.
\end{abstract}

MSC: 11B68; 11B83; 11580

Keywords: Euler polynomials and numbers; Degenerate Euler polynomials and numbers; Alternating integer power sum polynomials; Degenerate alternating integer power sum polynomials

\section{Introduction}

Let $p$ be a fixed odd prime number. Throughout this paper, $\mathbb{Z}_{p}, \mathbb{Q}_{p}$, and $\mathbb{C}_{p}$ will denote the ring of $p$-adic integers, the field of $p$-adic rational numbers, and the completion of the algebraic closure of $\mathbb{Q}_{p}$, respectively. The $p$-adic norm is normalized as $|p|_{p}=\frac{1}{p}$.

Let $f$ be a $\mathbb{C}_{p}$-valued continuous function on $\mathbb{Z}_{p}$. Then the fermionic $p$-adic integral of $f$ on $\mathbb{Z}_{p}$ is defined by Kim as

$$
\begin{aligned}
\int_{\mathbb{Z}_{p}} f(x) d \mu_{-1}(x) & =\lim _{N \rightarrow \infty} \sum_{x=0}^{p^{N}-1} f(x) \mu_{-1}\left(x+p^{N} \mathbb{Z}_{p}\right) \\
& =\lim _{N \rightarrow \infty} \sum_{x=0}^{p^{N}-1} f(x)(-1)^{x} \quad(\text { see }[11,12]) .
\end{aligned}
$$

From (1.1), we note that

$$
\int_{\mathbb{Z}_{p}} f(x+1) d \mu_{-1}(x)=-\int_{\mathbb{Z}_{p}} f(x) d \mu_{-1}(x)+2 f(0) \quad(\text { see }[5,8]),
$$

\section{Springer}


and by induction, for any $n \in \mathbb{N}$, we get

$$
\int_{\mathbb{Z}_{p}} f(x+n) d u_{-1}(x)=(-1)^{n} \int_{\mathbb{Z}_{p}} f(x) d u_{-1}(x)+2 \sum_{l=0}^{n-1}(-1)^{n-1-l} f(l) .
$$

It is well known that the Euler polynomials are defined by

$$
\frac{2}{e^{t}+1} e^{x t}=\sum_{n=0}^{\infty} E_{n}(x) \frac{t^{n}}{n !} \quad(\text { see }[1-21])
$$

When $x=0, E_{n}=E_{n}(0)$ are called the Euler numbers.

From (1.4), we note that

$$
E_{n}(x)=\sum_{l=0}^{n}\left(\begin{array}{l}
n \\
l
\end{array}\right) E_{l} x^{n-l}, \quad n \geq 0(\text { see }[1-21])
$$

where $n$ is a nonnegative integer.

By (1.4) and (1.5), we get

$$
E_{n}(1)+E_{n}=\sum_{l=0}^{n}\left(\begin{array}{l}
n \\
l
\end{array}\right) E_{l}+E_{n}= \begin{cases}2, & \text { if } n=0 \\
0, & \text { if } n>0\end{cases}
$$

Let

$$
T_{p}(n)=2 \sum_{k=1}^{n}(-1)^{k-1} k^{p}, \quad n, p \in \mathbb{N} .
$$

Then, by (1.4) and (1.5), we get

$$
\begin{aligned}
\sum_{p=0}^{\infty} T_{p}(n) \frac{t^{p}}{p !} & =2 \sum_{k=1}^{n}(-1)^{k-1} e^{k t}=\frac{2}{e^{t}+1}\left(e^{(n+1) t}+e^{t}\right) \\
& =\sum_{p=0}^{\infty}\left(E_{p}(n+1)+E_{p}(1)\right) \frac{t^{p}}{p !},
\end{aligned}
$$

where $n \in \mathbb{N}$, with $n \equiv 1(\bmod 2)$. Thus we have, for $n, p \in \mathbb{N}$, with $n \equiv 1(\bmod 2)$,

$$
T_{p}(n)=E_{p}(n+1)-E_{p}
$$

From (1.2), we can derive the following equation (1.10):

$$
\int_{\mathbb{Z}_{p}} e^{(x+y) t} d \mu_{-1}(y)=\frac{2}{e^{t}+1} e^{x t}=\sum_{n=0}^{\infty} E_{n}(x) \frac{t^{n}}{n !} .
$$

Thus, by (1.10), we get

$$
\int_{\mathbb{Z}_{p}}(x+y)^{n} d \mu_{-1}(y)=E_{n}(x), \quad n \geq 0(\text { see }[11]) .
$$


Thus, by (1.9) and (1.11), we have

$$
T_{p}(n)=\int_{\mathbb{Z}_{p}}(x+n+1)^{p} d \mu_{-1}(x)-\int_{\mathbb{Z}_{p}} x^{p} d \mu_{-1}(x),
$$

where $n, p \in \mathbb{N}$ with $n \equiv 1(\bmod 2)$.

We recall here that the Stirling numbers of the second kind are given by the exponential generating function

$$
\frac{1}{k !}\left(e^{t}-1\right)^{k}=\sum_{n=k}^{\infty} S_{2}(n, k) \frac{t^{n}}{n !}
$$

The purpose of this paper is to investigate some identities on Euler numbers and polynomials, and those on degenerate Euler numbers and polynomials which are derived from the fermionic $p$-adic integrals on $\mathbb{Z}_{p}$.

The outline of this paper is as follows. In Sect. 1, we will review some necessary results about fermionic $p$-adic integrals, Euler polynomials, and alternating integer power sums. In Sect. 2, we will introduce the alternating integer power sum polynomials and represent them in terms of Euler polynomials and Stirling numbers of the second kind, and derive various properties about Euler numbers and polynomials. In Sect. 3, we will introduce the degenerate alternating integer power sum polynomials and express them in terms of degenerate Euler polynomials and degenerate Stirling numbers of the second, and derive some properties on degenerate Euler numbers and polynomials.

\section{Some identities of Euler numbers and polynomials}

In this section, we will introduce the alternating integer power sum polynomials and represent them in terms of Euler polynomials and Stirling numbers of the second kind, and derive various properties about Euler numbers and polynomials.

For $p \in \mathbb{N}$, we have

$$
\begin{aligned}
(-1)^{j}(j+1)^{p}+(-1)^{j} j^{p} & =\sum_{i=0}^{p}\left(\begin{array}{c}
p \\
i
\end{array}\right) j^{i}(-1)^{j}+(-1)^{j} j^{p} \\
& =2(-1)^{j} j^{p}+\sum_{i=1}^{p-1}\left(\begin{array}{c}
p \\
i
\end{array}\right) j^{i}(-1)^{j}+(-1)^{j} .
\end{aligned}
$$

From (2.1), for $n, p \in \mathbb{N}$ with $n \equiv 1(\bmod 2)$, we note that

$$
\begin{aligned}
(-1)^{n}(n+1)^{p} & =\sum_{j=0}^{n}\left\{(-1)^{j}(j+1)^{p}+(-1)^{j} j^{p}\right\} \\
& =2 \sum_{j=0}^{n}(-1)^{j} j^{p}+\sum_{i=1}^{p-1}\left(\begin{array}{c}
p \\
i
\end{array}\right) \sum_{j=0}^{n}(-1)^{j} j^{i}+\sum_{j=0}^{n}(-1)^{j} \\
& =2 \sum_{j=1}^{n}(-1)^{j} j^{p}+\sum_{i=1}^{p-1}\left(\begin{array}{c}
p \\
i
\end{array}\right) \sum_{j=1}^{n}(-1)^{j} j^{i} .
\end{aligned}
$$


By (1.7) and (2.2), we get

$$
T_{p}(n)=(n+1)^{p}-\frac{1}{2} \sum_{i=1}^{p-1}\left(\begin{array}{c}
p \\
i
\end{array}\right) T_{i}(n)
$$

where $n, p \in \mathbb{N}$ with $n \equiv 1(\bmod 2)$.

Therefore, by (2.3), we obtain the following theorem.

Theorem 2.1 Let $n, p \in \mathbb{N}$ with $n \equiv 1(\bmod 2)$. Then we have

$$
\begin{aligned}
& \int_{\mathbb{Z}_{p}}(x+n+1)^{p} d \mu_{-1}(x)-\int_{\mathbb{Z}_{p}} x^{p} d \mu_{-1}(x) \\
& =(n+1)^{p}-\frac{1}{2} \sum_{i=1}^{p-1}\left(\begin{array}{c}
p \\
i
\end{array}\right)\left\{\int_{\mathbb{Z}_{p}}(x+n+1)^{i} d \mu_{-1}(x)-\int_{\mathbb{Z}_{p}} x^{i} d \mu_{-1}(x)\right\} .
\end{aligned}
$$

From (1.11) and Theorem 2.1, we note the following corollary.

Corollary 2.2 Let $n, p \in \mathbb{N}$ with $n \equiv 1(\bmod 2)$. Then we have

$$
E_{p}(n+1)-E_{p}=(n+1)^{p}-\frac{1}{2} \sum_{i=1}^{p-1}\left(\begin{array}{l}
p \\
i
\end{array}\right)\left(E_{i}(n+1)-E_{i}\right) .
$$

For $n \in \mathbb{N}_{0}=\mathbb{N} \cup\{0\}$, we have

$$
\int_{\mathbb{Z}_{p}}(y+1-x)^{n} d \mu_{-1}(y)=(-1)^{n} \int_{\mathbb{Z}_{p}}(y+x)^{n} d \mu_{-1}(y) .
$$

Thus, by (2.6), we get

$$
E_{n}(1-x)=(-1)^{n} E_{n}(x), \quad n \geq 0 .
$$

For $n \in \mathbb{N}_{0}$, and by (1.2), we have

$$
\begin{aligned}
\int_{\mathbb{Z}_{p}}(x+2)^{n} d \mu_{-1}(x) & =\sum_{l=0}^{n}\left(\begin{array}{l}
n \\
l
\end{array}\right) \int_{\mathbb{Z}_{p}}(x+1)^{l} d \mu_{-1}(x) \\
& =1+\sum_{l=1}^{n}\left(\begin{array}{l}
n \\
l
\end{array}\right) \int_{\mathbb{Z}_{p}}(x+1)^{l} d \mu_{-1}(x) \\
& =1-\sum_{l=1}^{n}\left(\begin{array}{l}
n \\
l
\end{array}\right) \int_{\mathbb{Z}_{p}} x^{l} d \mu_{-1}(x) \\
& =2-\sum_{l=0}^{n}\left(\begin{array}{l}
n \\
l
\end{array}\right) \int_{\mathbb{Z}_{p}} x^{l} d \mu_{-1}(x) .
\end{aligned}
$$

Thus, by using (1.2) and (2.7), we get the next theorem. 
Theorem 2.3 For $n \in \mathbb{N} \cup\{0\}$, we have

$$
\int_{\mathbb{Z}_{p}}(x+2)^{n} d \mu_{-1}(x)=2+\int_{\mathbb{Z}_{p}} x^{n} d \mu_{-1}(x)-2 \delta_{0, n},
$$

where $\delta_{n, k}$ is the Kronecker's delta.

By combining Theorem 2.3 with (1.11), we arrive at the following corollary.

Corollary 2.4 For $n \in \mathbb{N}$, we have

$$
E_{n}(2)=2+E_{n}
$$

For the next result, we note that, for any $n \in \mathbb{N}$,

$$
E_{n}=(-1)^{n-1} E_{n}
$$

For $m, n \in \mathbb{N}$, and by (1.11) and (2.9), we have

$$
\begin{aligned}
\int_{\mathbb{Z}_{p}} x^{m}(x-1)^{n} d \mu_{-1}(x) & =\sum_{i=0}^{n}\left(\begin{array}{l}
n \\
i
\end{array}\right)(-1)^{n-i} \int_{\mathbb{Z}_{p}} x^{m+i} d \mu_{-1}(x) \\
& =\sum_{i=0}^{n}\left(\begin{array}{l}
n \\
i
\end{array}\right)(-1)^{n-i} E_{m+i} \\
& =(-1)^{m+n-1} \sum_{i=0}^{n}\left(\begin{array}{l}
n \\
i
\end{array}\right) E_{m+i} .
\end{aligned}
$$

On the other hand, by (2.6) and (2.8), we get

$$
\begin{aligned}
\int_{\mathbb{Z}_{p}} x^{m}(x-1)^{n} d \mu_{-1}(x) & =\sum_{i=0}^{m}\left(\begin{array}{c}
m \\
i
\end{array}\right) \int_{\mathbb{Z}_{p}}(x-1)^{n+i} d \mu_{-1}(x) \\
& =\sum_{i=0}^{m}\left(\begin{array}{c}
m \\
i
\end{array}\right)(-1)^{n+i} \int_{\mathbb{Z}_{p}}(x+2)^{n+i} d \mu_{-1}(x) \\
& =\sum_{i=0}^{m}\left(\begin{array}{c}
m \\
i
\end{array}\right)(-1)^{n+i}\left(E_{n+i}+2\right) \\
& =\sum_{i=0}^{m}\left(\begin{array}{c}
m \\
i
\end{array}\right)(-1)^{n+i} E_{n+i} \\
& =-\sum_{i=0}^{m}\left(\begin{array}{c}
m \\
i
\end{array}\right) E_{n+i} .
\end{aligned}
$$

Therefore, by (2.10) and (2.11), we obtain the following theorem.

Theorem 2.5 For $m, n \in \mathbb{N}$, the following symmetric identity holds:

$$
(-1)^{n} \sum_{i=0}^{n}\left(\begin{array}{c}
n \\
i
\end{array}\right) E_{m+i}=(-1)^{m} \sum_{i=0}^{m}\left(\begin{array}{c}
m \\
i
\end{array}\right) E_{n+i}
$$


Now, we define the alternating integer power sum polynomials by

$$
T_{p}(n \mid x)=2 \sum_{k=0}^{n}(-1)^{k-1}(k+x)^{p}, \quad n, p \in \mathbb{N}_{0}
$$

Note that $T_{p}(n \mid 0)=T_{p}(n), n \in \mathbb{N}_{0}, p \in \mathbb{N}$.

For $N \in \mathbb{N}$ with $N \equiv 1(\bmod 2)$, by $(1.3)$, we get

$$
\begin{aligned}
& 2 \sum_{k=0}^{N}(-1)^{k-1} e^{(k+x) t} \\
& \quad=\int_{\mathbb{Z}_{p}} e^{(N+1+x+y) t} d \mu_{-1}(y)-\int_{\mathbb{Z}_{p}} e^{(x+y) t} d \mu_{-1}(y) \\
& \quad=\sum_{n=0}^{\infty}\left\{\int_{\mathbb{Z}_{p}}(N+1+x+y)^{n} d \mu_{-1}(y)-\int_{\mathbb{Z}_{p}}(x+y)^{n} d \mu_{-1}(y)\right\} \frac{t^{n}}{n !} .
\end{aligned}
$$

Now, we see that (2.14) is equivalent to the next theorem.

Theorem 2.6 For $N \in \mathbb{N}$, with $N \equiv 1(\bmod 2)$, and $n \in \mathbb{N}_{0}$, we have

$$
T_{n}(N \mid x)=E_{n}(x+N+1)-E_{n}(x) .
$$

From (2.14), and recalling (1.13), we note that

$$
\begin{aligned}
2 \sum_{k=0}^{N}(-1)^{k-1} e^{(k+x) t} & =e^{x t}\left(e^{(N+1) t}-1\right) \int_{\mathbb{Z}_{p}} e^{y t} d \mu_{-1}(y) \\
& =\frac{2}{e^{t}+1} e^{x t}\left(\sum_{m=0}^{N+1}\left(\begin{array}{c}
N+1 \\
m
\end{array}\right)\left(e^{t}-1\right)^{m}-1\right) \\
& =\left(\sum_{j=0}^{\infty} E_{j}(x) \frac{t^{j}}{j !}\right)\left(\sum_{l=0}^{\infty}\left(\sum_{m=0}^{l}\left(\begin{array}{c}
N+1 \\
m
\end{array}\right) m ! S_{2}(l, m)\right) \frac{t^{l}}{l !}-1\right) \\
& =\sum_{n=0}^{\infty}\left\{\sum_{l=0}^{n} \sum_{m=0}^{l}\left(\begin{array}{c}
N+1 \\
m
\end{array}\right)\left(\begin{array}{c}
n \\
l
\end{array}\right) m ! S_{2}(l, m) E_{n-l}(x)-E_{n}(x)\right\} \frac{t^{n}}{n !},
\end{aligned}
$$

where $N \in \mathbb{N}$ with $N \equiv 1(\bmod 2)$ and $S_{2}(l, m)$ is the Stirling number of the second kind.

Therefore, by (2.16), we obtain the following theorem.

Theorem 2.7 For $N \in \mathbb{N}$ with $N \equiv 1(\bmod 2)$ and $n \in \mathbb{N}_{0}$, we have

$$
T_{n}(N \mid x)=\sum_{l=0}^{n} \sum_{m=0}^{l}\left(\begin{array}{c}
N+1 \\
m
\end{array}\right)\left(\begin{array}{l}
n \\
l
\end{array}\right) m ! S_{2}(l, m) E_{n-l}(x)-E_{n}(x)
$$

where $S_{2}(n, m)$ is the Stirling number of the second kind. 
For $m, k \in \mathbb{N}$ with $m-k \geq 1$, and making use of (1.2) and (2.9), we have

$$
\begin{aligned}
& (-1)^{m-k} \int_{\mathbb{Z}_{p}} x^{m-k} d \mu_{-1}(x) \\
& =-\int_{\mathbb{Z}_{p}} x^{m-k} d \mu_{-1}(x)=\int_{\mathbb{Z}_{p}}(x+1)^{m-k} d \mu_{-1}(x) \\
& =\sum_{j=0}^{m-k}\left(\begin{array}{c}
m-k \\
m-k-j
\end{array}\right) \int_{\mathbb{Z}_{p}} x^{m-k-j} d \mu_{-1}(x)=\sum_{j=k}^{m}\left(\begin{array}{l}
m-k \\
m-j
\end{array}\right) \int_{\mathbb{Z}_{p}} x^{m-j} d \mu_{-1}(x) \\
& =\frac{1}{\left(\begin{array}{c}
m \\
k
\end{array}\right)} \sum_{j=k}^{m}\left(\begin{array}{c}
m \\
j
\end{array}\right)\left(\begin{array}{l}
j \\
k
\end{array}\right) \int_{\mathbb{Z}_{p}} x^{m-j} d \mu_{-1}(x) .
\end{aligned}
$$

Theorem 2.8 For $m, k \in \mathbb{N}$ with $m-k \geq 1$, we have

$$
(-1)^{m-k}\left(\begin{array}{c}
m \\
k
\end{array}\right) E_{m-k}=\sum_{j=k}^{m}\left(\begin{array}{c}
m \\
j
\end{array}\right)\left(\begin{array}{l}
j \\
k
\end{array}\right) E_{m-j}
$$

\section{Some identities of degenerate Euler numbers and polynomials}

In this section, we will introduce the degenerate alternating integer power sum polynomials and express them in terms of degenerate Euler polynomials and degenerate Stirling numbers of the second, and derive some properties on degenerate Euler numbers and polynomials.

Throughout this section, we assume that $\lambda \in \mathbb{C}_{p}$ with $|\lambda|_{p}<p^{-\frac{1}{p-1}}$. The degenerate exponential function is defined as

$$
e_{\lambda}^{x}(t)=(1+\lambda t)^{\frac{x}{\lambda}}, \quad e_{\lambda}(t)=e_{\lambda}^{1}(t), \quad n \geq 0(\text { see }[3,4,14-16])
$$

Note that $\lim _{\lambda \rightarrow 0} e_{\lambda}^{x}(t)=e^{x t}$.

It is well known that the degenerate Euler polynomials are defined by L. Carlitz as

$$
\frac{2}{e_{\lambda}(t)+1} e_{\lambda}^{x}(t)=\frac{2}{(1+\lambda t)^{\frac{1}{\lambda}}+1}(1+\lambda t)^{\frac{x}{\lambda}}=\sum_{n=0}^{\infty} \mathcal{E}_{n, \lambda}(x) \frac{t^{n}}{n !} .
$$

When $x=0, \mathcal{E}_{n, \lambda}=\mathcal{E}_{n, \lambda}(0)$ are called the degenerate Euler numbers (see [3, 4, 14-17]).

From (3.1), we note that

$$
\mathcal{E}_{n, \lambda}(x)=\sum_{l=0}^{n}\left(\begin{array}{l}
n \\
l
\end{array}\right)(x)_{n-l, \lambda} \mathcal{E}_{l, \lambda}, \quad n \geq 0(\text { see }[3,4,14-17])
$$

where $(x)_{n, \lambda}=x(x-\lambda) \cdots(x-(n-1) \lambda), n \geq 1,(x)_{0, \lambda}=1$.

From (3.1), we can derive the following recurrence relation for $\mathcal{E}_{n, \lambda}, n \geq 0$.

$$
\sum_{l=0}^{n}\left(\begin{array}{l}
n \\
l
\end{array}\right)(1)_{n-l, \lambda} \mathcal{E}_{l, \lambda}+\mathcal{E}_{n, \lambda}= \begin{cases}2, & \text { if } n=0 \\
0, & \text { if } n>0\end{cases}
$$


From (3.2) and (3.3), we have

$$
\mathcal{E}_{n, \lambda}(1)=-\mathcal{E}_{n, \lambda}+2 \delta_{0, n}, \quad n \geq 0 .
$$

For $N \in \mathbb{N}$ with $N \equiv 1(\bmod 2)$, we have

$$
\begin{aligned}
2 \sum_{k=0}^{N}(-1)^{k-1} e_{\lambda}^{k+x}(t) & =\frac{2}{e_{\lambda}(t)+1}\left(e_{\lambda}^{N+1+x}(t)-e_{\lambda}^{x}(t)\right) \\
& =\sum_{n=0}^{\infty}\left\{\mathcal{E}_{n, \lambda}(N+1+x)-\mathcal{E}_{n, \lambda}(x)\right\} \frac{t^{n}}{n !}
\end{aligned}
$$

On the other hand,

$$
2 \sum_{k=0}^{N}(-1)^{k-1} e_{\lambda}^{k+x}(t)=\sum_{n=0}^{\infty}\left(2 \sum_{k=0}^{N}(-1)^{k-1}(k+x)_{n, \lambda}\right) \frac{t^{n}}{n !}
$$

Let us define a degenerate version of the alternating integer power sum polynomials, called the degenerate alternating integer power sum polynomials, by

$$
T_{p, \lambda}(n \mid x)=2 \sum_{k=0}^{n}(-1)^{k-1}(k+x)_{p, \lambda}, \quad n \geq 0 .
$$

Note that $\lim _{\lambda \rightarrow 0} T_{p, \lambda}(n \mid x)=T_{p}(n \mid x), n \geq 0$.

Therefore, by (3.5) and (3.6), we obtain the following theorem.

Theorem 3.1 For $n \in \mathbb{N}_{0}$, and $N \in \mathbb{N}$, with $N \equiv 1(\bmod 2)$, we have

$$
T_{n, \lambda}(N \mid x)=\mathcal{E}_{n, \lambda}(N+1+x)-\mathcal{E}_{n, \lambda}(x)
$$

From (1.2), we note that

$$
\int_{\mathbb{Z}_{p}} e_{\lambda}^{x+y}(t) d \mu_{-1}(y)=\frac{2}{e_{\lambda}(t)+1} e_{\lambda}^{x}(t)=\sum_{n=0}^{\infty} \mathcal{E}_{n, \lambda}(x) \frac{t^{n}}{n !} .
$$

On the other hand,

$$
\int_{\mathbb{Z}_{p}} e_{\lambda}^{x+y}(t) d \mu_{-1}(y)=\sum_{n=0}^{\infty} \int_{\mathbb{Z}_{p}}(x+y)_{n, \lambda} d \mu_{-1}(y) \frac{t^{n}}{n !} .
$$

By (3.7) and (3.8), we get

$$
\int_{\mathbb{Z}_{p}}(x+y)_{n, \lambda} d \mu_{-1}(y)=\mathcal{E}_{n, \lambda}(x), \quad n \geq 0 .
$$


For $d \in \mathbb{N}$ with $d \equiv 1(\bmod 2)$, by (1.3), we get

$$
\begin{aligned}
& \int_{\mathbb{Z}_{p}} e_{\lambda}^{x+y}(t) d \mu_{-1}(y) \\
& =\frac{2}{e_{\lambda}^{d}(t)+1} \sum_{l=0}^{d-1}(-1)^{l} e_{\lambda}^{x+l}(t) \\
& =\frac{2}{e_{\frac{\lambda}{d}}(d t)+1} \sum_{l=0}^{d-1}(-1)^{l} e_{\frac{\lambda}{d}}^{\frac{x+l}{d}}(d t)=\sum_{l=0}^{d-1}(-1)^{l} \sum_{n=0}^{\infty} \mathcal{E}_{n, \frac{\lambda}{d}}\left(\frac{x+l}{d}\right) \frac{d^{n} t^{n}}{n !} \\
& =\sum_{n=0}^{\infty}\left(d^{n} \sum_{l=0}^{d-1}(-1)^{l} \mathcal{E}_{n, \frac{\lambda}{d}}\left(\frac{x+l}{d}\right)\right) \frac{t^{n}}{n !} .
\end{aligned}
$$

From (3.9) and (3.10), we have

$$
\mathcal{E}_{n, \lambda}(x)=d^{n} \sum_{l=0}^{d-1}(-1)^{l} \mathcal{E}_{n, \frac{\lambda}{d}}\left(\frac{x+l}{d}\right)
$$

where $n \in \mathbb{N}_{0}$ and $d \in \mathbb{N}$ with $d \equiv 1(\bmod 2)$.

From (3.7) and (3.9), we have

$$
\int_{\mathbb{Z}_{p}}(1-x+y)_{n, \lambda} d \mu_{-1}(y)=(-1)^{n} \int_{\mathbb{Z}_{p}}(x+y)_{n,-\lambda} d \mu_{-1}(y),
$$

where $n$ is a nonnegative integer.

Hence, by (3.7), we get

$$
\mathcal{E}_{n, \lambda}(1-x)=(-1)^{n} \mathcal{E}_{n,-\lambda}(x), \quad n \geq 0 .
$$

Now, we observe that

$$
\begin{aligned}
2 \sum_{k=0}^{N}(-1)^{k-1} e_{\lambda}^{x+k}(t) & =\frac{2}{e_{\lambda}(t)+1}\left(e_{\lambda}^{N+1+x}(t)-e_{\lambda}^{x}(t)\right) \\
& =\frac{2}{e_{\lambda}(t)+1}\left(\left(e_{\lambda}(t)-1+1\right)^{N+1}-1\right) e_{\lambda}^{x}(t) \\
& =\frac{2}{e_{\lambda}(t)+1}\left(\sum_{m=0}^{N+1}\left(\begin{array}{c}
N+1 \\
m
\end{array}\right)\left(e_{\lambda}(t)-1\right)^{m}-1\right) e_{\lambda}^{x}(t),
\end{aligned}
$$

where $N \in \mathbb{N}$, with $N \equiv 1(\bmod 2)$.

As is well known, the degenerate Stirling numbers of the second kind are given by the generating function as

$$
\frac{1}{k !}\left(e_{\lambda}(t)-1\right)^{k}=\sum_{n=k}^{\infty} S_{2, \lambda}(n, k) \frac{t^{n}}{n !} \quad(\text { see }[14,16,18])
$$


From (3.14) and (3.15), we have

$$
\begin{aligned}
2 \sum_{k=0}^{N}(-1)^{k-1} e_{\lambda}^{x+k}(t) & =\sum_{j=0}^{\infty} \mathcal{E}_{j, \lambda}(x) \frac{t^{j}}{j !}\left(\sum_{l=0}^{\infty} \sum_{m=0}^{l}\left(\begin{array}{c}
N+1 \\
m
\end{array}\right) m ! S_{2, \lambda}(l, m) \frac{t^{l}}{l !}-1\right) \\
& =\sum_{n=1}^{\infty}\left(\sum_{l=1}^{n} \sum_{m=1}^{l}\left(\begin{array}{c}
n \\
l
\end{array}\right)\left(\begin{array}{c}
N+1 \\
m
\end{array}\right) m ! \mathcal{E}_{n-l, \lambda}(x) S_{2, \lambda}(l, m)\right) \frac{t^{n}}{n !}
\end{aligned}
$$

The left-hand side of (3.16) is given by

$$
\begin{aligned}
2 \sum_{k=0}^{N}(-1)^{k-1} e_{\lambda}^{x+k}(t) & =\sum_{n=0}^{\infty}\left(2 \sum_{k=0}^{N}(-1)^{k-1}(x+k)_{n, \lambda}\right) \frac{t^{n}}{n !} \\
& =\sum_{n=1}^{\infty} T_{n, \lambda}(N \mid x) \frac{t^{n}}{n !}
\end{aligned}
$$

where $N \in \mathbb{N}$ with $N \equiv 1(\bmod 2)$.

Therefore, by (3.16) and (3.17), we obtain the following theorem.

Theorem 3.2 For $n, N \in \mathbb{N}$, with $N \equiv 1(\bmod 2)$, we have

$$
T_{n, \lambda}(N \mid x)=\sum_{l=1}^{n} \sum_{m=1}^{l}\left(\begin{array}{l}
n \\
l
\end{array}\right)\left(\begin{array}{c}
N+1 \\
m
\end{array}\right) m ! \mathcal{E}_{n-l, \lambda}(x) S_{2, \lambda}(l, m) .
$$

\section{Conclusions}

As is well known, the alternating integer power sums can be expressed in terms of some values of Euler polynomials. In this paper, we studied some identities on Euler numbers and polynomials, and those on degenerate Euler numbers and polynomials, which are derived from certain fermionic $p$-adic integrals on $\mathbb{Z}_{p}$. Here we mention that fermionic $p$-adic integrals were introduced by Kim and have been used fruitfully in investigations of combinatorial and number-theoretic aspects of many special numbers and polynomials.

Specifically, we obtained a recursive formula for alternating integer power sums and representations of alternating integer power sum polynomials in terms of Euler polynomials and also of Euler polynomials together with Stirling numbers of the second kind. Along the way, various properties of Euler numbers and polynomials were derived as well. As to the degenerate alternating integer power sum polynomials associated with the alternating integer power sums, we obtained their representations in terms of degenerate Euler polynomials and also of degenerate Euler polynomials together with the degenerate Stirling numbers of the second kind. Along the way, we also derived some properties of degenerate Euler numbers and polynomials.

Acknowledgements

We would like to thank the referees for their valuable comments and suggestions that improved the original manuscript in its present form.

Funding

This research received no external funding. 
Authors' contributions

Each of the authors TK, DSK, HYK, and JK contributed to each part of this study equally, as well as read and approved the final version of the manuscript.

\section{Author details}

'School of Science, Xian Technological University, Xian, China. ${ }^{2}$ Department of Mathematics, Kwangwoon University, Seoul, Republic of Korea. ${ }^{3}$ Department of Mathematics, Sogang University, Seoul, Republic of Korea. ${ }^{4}$ Department of Mathematics Education and ERI, Gyeongsang National University, Jinju, Republic of Korea.

\section{Publisher's Note}

Springer Nature remains neutral with regard to jurisdictional claims in published maps and institutional affiliations.

Received: 29 July 2019 Accepted: 11 October 2019 Published online: 17 October 2019

\section{References}

1. Araci, S., Acikgoz, M.: A note on the Frobenius-Euler numbers and polynomials associated with Bernstein polynomials. Adv. Stud. Contemp. Math. (Kyungshang) 22(3), 399-406 (2012)

2. Bayad, A., Chikhi, J.: Apostol-Euler polynomials and asymptotics for negative binomial reciprocals. Adv. Stud. Contemp. Math. (Kyungshang) 24(1), 33-37 (2014)

3. Carlitz, L.: A degenerate Staudt-Clausen theorem. Arch. Math. (Basel) 7, 28-33 (1956)

4. Carlitz, L.: Degenerate Stirling, Bernoulli and Eulerian numbers. Util. Math. 15, 51-88 (1979)

5. Dolgy, D.V., Kim, D.S., Kwon, J., Kim, T.: Some identities of ordinary and degenerate Bernoulli numbers and polynomials. Symmetry 11(7), 847 (2019)

6. Kim, D.S.: Identities associated with generalized twisted Euler polynomials twisted by ramified roots of unity. Adv Stud. Contemp. Math. (Kyungshang) 22(3), 363-377 (2012)

7. Kim, D.S., Dolgy, D.V., Kim, T., Kim, D.: Extended degenerate $r$-central factorial numbers of the second kind and extended degenerate $r$-central Bell polynomials. Symmetry 11(4), 595 (2019)

8. Kim, D.S., Dolgy, D.V., Kwon, J., Kim, T.: Note on type 2 degenerate q-Bernoulli polynomials. Symmetry 11(7), 914 (2019)

9. Kim, D.S., Kim, H.Y., Kim, D., Kim, T.: Identities of symmetry for type 2 Bernoulli and Euler polynomials. Symmetry 11(5), $613(2019)$

10. Kim, D.S., Lee, N., Na, J., Park, K.H.: Identities of symmetry for higher-order Euler polynomials in three variables (I). Adv. Stud. Contemp. Math. (Kyungshang) 22(1), 51-74 (2012)

11. Kim, T.: $q$-Euler numbers and polynomials associated with $p$-adic $q$-integrals. J. Nonlinear Math. Phys. 14(1), 15-27 (2007)

12. Kim, T.: New approach to q-Euler polynomials of higher order. Russ. J. Math. Phys. 17(2), 218-225 (2010)

13. Kim, T., Kim, D.S.: Degenerate Laplace transform and degenerate gamma function. Russ. J. Math. Phys. 24(2), 241-248 (2017)

14. Kim, T., Kim, D.S.: Degenerate Bernstein polynomials. Rev. R. Acad. Cienc. Exactas Fís. Nat., Ser. A Mat. 113(3) 2913-2920 (2019)

15. Kim, T., Kim, D.S.: A note on type 2 Changhee and Daehee polynomials. Rev. R. Acad. Cienc. Exactas Fís. Nat., Ser. A Mat. 113(3), 2763-2771 (2019)

16. Kim, T. Kim, D.S.: Degenerate central Bell numbers and polynomials. Rev. R. Acad. Cienc. Exactas Fís. Nat., Ser. A Mat. 113(3), 2507-2513 (2019)

17. Kim, T., Kim, D.S., Jang, G.-W., Kwon, J.: A note on degenerate Bernstein polynomials. J. Inequal. Appl. 2019, 129, 12 pp. (2019)

18. Kim, W.J., Kim, D.S., Kim, H.Y., Kim, T.: Some identities of degenerate Euler polynomials associated with degenerate Bernstein polynomials. J. Inequal. Appl. 2019, 160, 11 pp. (2019)

19. Rim, S.-H., Jeong, J.: On the modified $q$-Euler numbers of higher order with weight. Adv. Stud. Contemp. Math. (Kyungshang) 22(1), 93-98 (2012)

20. Sen, E.: Theorems on Apostol-Euler polynomials of higher order arising from Euler basis. Adv. Stud. Contemp. Math. (Kyungshang) 23(2), 337-345 (2013)

21. Simsek, Y.: Identities and relations related to combinatorial numbers and polynomials. Proc. Jangjeon Math. Soc 20(1), 127-135 (2017) 